\title{
Modélisation du tassement sous poids propre des couches de vase molle et saturée, sur un fond imperméable
}

\author{
Martin Sanchez, Alain Grovel \\ Laboratoire de mécanique et géomécanique de l'Université de Nantés \\ 2, rue de la Houssinière, 44072 Nantes Cedex 03
}

\section{Introduction}

Le tassement des vases complètement saturées peut être subdivisé en deux processus (fig. 1 ) :

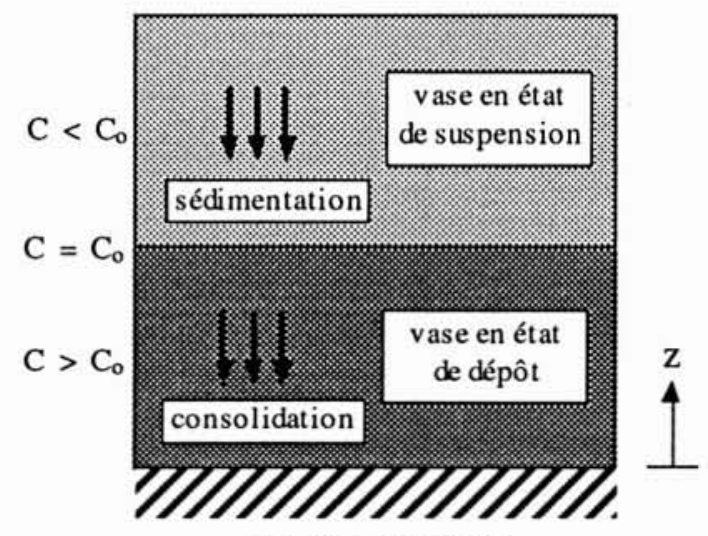

Fond imperméable

1. Diagramme illustrant les processus de tassement avec lesétats associés de la vase.

a) Sédimentation, lorsque les particules élémentaires de la vase ne forment un réseau continu tridimensionnel qu'à l'échelle de la dimension des flocons. Dans ce cas, la vase est en état de suspension.

b) Consolidation, lorsque les particules élémentaires de la vase forment un réseau continu tridimensionnel. Dans ce cas, caractérisé par la présence des contraintes effectives, une partie des contraintes est transmise grain à grain et donc, la vase est en état de dépôt.

A température constante, l'état d'une vase donnée dépend de sa concentration, laquelle est la variable d'état de la vase dans ce qui suit.

Ainsi, à partir d'essais de tassement réalisés en laboratoire sur des boues d'origines très diverses, CARRIER et al. [2] déterminent que pour des buts pratiques, on peut considérer que la limite entre les phénomènes de sédimentation et de consolidation correspond à une teneur en eau égale à 7 fois la limite de liquidité du matériau, soit une concentration $C_{0}$ donnée par :

$$
C_{0}=\frac{\rho_{0}}{\frac{\rho_{0}}{\rho_{s}}+\frac{7 W_{L}}{100}}
$$

où $C_{0}$ est la concentration frontière entre les états de dépôt et de suspension $\left(\mathrm{kg} \mathrm{m}^{-3}\right), \rho_{0}$ la masse volumique de la phase fluide $\left(\mathrm{kg} \mathrm{m}^{-3}\right), \rho_{s}$ la masse volumique de la phase solide $\left(\mathrm{kg} \mathrm{m}^{-3}\right)$ et $W_{L}$ la limite de liquidité (teneur en eau en \%).

Indépendamment de son état, l'évolution de la concentration dans une couche de vase est régie par l'équation de continuité de la phase solide :

$$
\frac{\partial C}{\partial t}+\frac{\partial\left[\bar{w}_{s} C\right]}{\partial z}=0
$$

où $C$ est la concentration de la suspension $\left(\mathrm{kg} \mathrm{m}^{-3}\right)$, $\bar{w}_{s}$ la vitesse moyenne de la phase solide $\left(\mathrm{m} \mathrm{s}^{-1}\right), t$ le temps $(s)$, et $z$ la coordonnée verticale $(m)$. 


\section{Vitesse moyenne de sédimentation des vases}

La vitesse moyenne de sédimentation des vases correspond à la vitesse moyenne de la phase solide dans le cas d'une suspension. Elle est définie comme étant le quotient du débit volumique de la phase solide par la surface occupée par la propre phase solide. La vitesse de sédimentation ainsi définie est donc la vitesse moyenne des particules solides.

En milieu aqueux floculant, les particules élémentaires des sédiments cohésifs forment des flocons ayant une vitesse de chute nettement supérieure à celle des particules élémentaires.

D'une manière générale, la floculation est d'autant plus importante que le milieu aqueux contient davantage de sels floculants, que les particules élémentaires sont plus petites, et que la concentration est plus élevée (MIGNIOT [7]).

Pour un matériau donné et un milieu aqueux donné, la vitesse moyenne de sédimentation sous conditions d'eau calme dépend essentiellement de la concentration (KYNCH [5] et THORN [13]).

Pour des concentrations faibles, inférieures à une concentration $C_{u}$ d'environ $0,3 \mathrm{~kg} \mathrm{~m}^{-3}$, Mehta [6] montre que la vitesse de sédimentation des matériaux cohésifs est pratiquement constante.

Pour des concentrations plus élevées, la floculation et la vitesse de sédimentation augmentent avec la concentration. Cependant, pour des concentrations supérieures à environ $10 \mathrm{~kg} \mathrm{~m}^{-3}$, on observe une diminution de la vitesse de sédimentation (vitesse entravée) [7].

La courbe caractéristique reliant $\bar{w}_{s}$ à la concentration d'une vase en état de suspension est approchée par l'équation suivante [9] (fig. 2) :

$$
\begin{aligned}
& \bar{w}_{s}=-\frac{k^{\prime}}{\rho_{0}}\left(1-\frac{\rho_{0}}{\rho_{s}}\right) C, \\
& \quad \text { valable pour : } C_{u}<C<C_{0} \\
& \text { avec : } \quad k^{\prime}=A_{3} \exp \left(-A_{4} \frac{C}{\rho_{s}}\right)
\end{aligned}
$$

où $A_{3}\left(\mathrm{~m} \mathrm{~s}^{-1}\right)$ et $A_{4}$ sont des paramètres propres à chaque vase et $\mathbf{k}^{\prime}\left(\mathrm{m} \mathrm{s}^{-1}\right)$ un coefficient qui, lorsque la concentration est égale à $C_{0}$, s'identifie au coefficient de perméabilité selon la définition de la loi de Darcy modifiée par Scheiddeger [10].

D'après la formulation précédente, le maximum de la vitesse moyenne correspond à une concentration $C_{\bar{w}_{s \max }}$ donnée par:

$$
C_{\bar{w}_{s \max }}=\rho_{s} / A_{4} .
$$

La vitesse de sédimentation maximale $\bar{w}_{s \max }$, associée à la concentration $C_{\bar{w}_{s \max }}$ est :

$$
\bar{w}_{s \max }=-A_{3} \exp (-1) \frac{1}{\rho_{0}}\left(1-\frac{\rho_{0}}{\rho_{s}}\right) \frac{\rho_{s}}{A_{4}} .
$$

La masse volumique de la phase solide des vases vaut environ $2550 \mathrm{~kg} \mathrm{~m}^{-3}$ et $C_{\bar{w}_{s \max }} \approx 10 \mathrm{~kg} \mathrm{~m}^{-3}$, ce qui conduit d'après l'équation (5) à une valeur typique pour $A_{4}$ de 255.

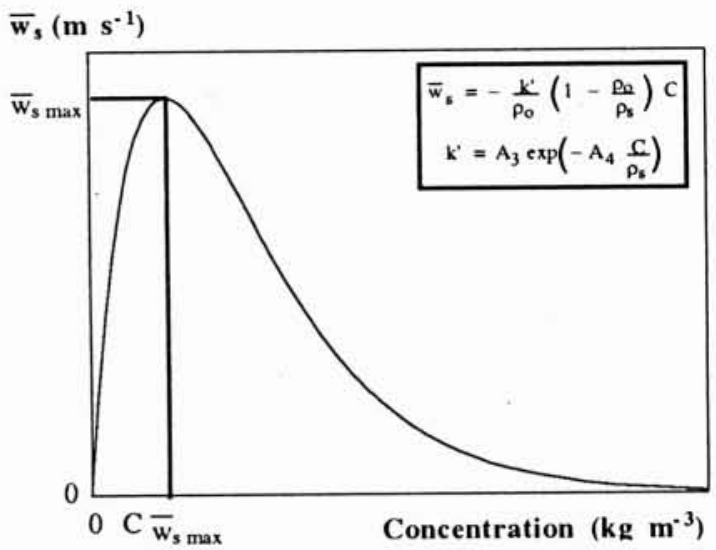

2. Courbe caractéristique reliant la vitesse de sédimentation (en valeur absolue sur le graphique) à la concentration d'une suspension (3) et (4).

\section{Vitesse de la phase solide dans les dépôts de vase}

Dans le cas des dépôts de vase, la vitesse moyenne de la phase fluide est reliée au gradient de surpression interstitielle par la loi de Darcy modifiée par SCHEIDEGGER [10] pour tenir compte de l'influence de la vitesse de la phase solide sur l'écoulement de la phase liquide :

$$
\left(1-\frac{C}{\rho_{s}}\right)\left(\bar{w}_{f}-\bar{w}_{s}\right)=-k \frac{1}{\rho_{0} g} \frac{\partial u}{\partial z}
$$

où $\bar{w}_{f}$ est la vitesse moyenne de la phase fluide $\left(\mathrm{m} \mathrm{s}^{-1}\right), k$ le coefficient de perméabilité $\left(\mathrm{m} \mathrm{s}^{-1}\right), g$ l'accélération de la pesanteur $\left(\mathrm{m} \mathrm{s}^{-2}\right)$ et $u$ la surpression interstitielle $(\mathrm{Pa})$.

Si la phase solide et la phase fluide sont incompressibles, l'équation de la continuité simultanée des deux phases est :

$$
\frac{\partial}{\partial z}\left[\bar{w}_{s} \frac{C}{\rho_{s}}+\bar{w}_{f}\left(1-\frac{C}{\rho_{s}}\right)\right]=0 .
$$

En intégrant (8) et en combinant avec (7), on obtient l'expression qui donne $\bar{w}_{s}$ en fonction de $\partial u / \partial z$, pour le cas d'un fond imperméable, ou plus généralement pour un drainage exclusivement superficiel :

$$
\bar{w}_{s}=k \frac{1}{\rho_{0} g} \frac{\partial u}{\partial z} .
$$

D'autre part, en tout moment, la condition d'équilibre dans un dépôt s'écrit :

$$
u=\sigma-\sigma^{\prime}
$$

où $\sigma$ est la contrainte appliquée due au poids déjaugé de la propre vase $(\mathrm{Pa})$ et $\sigma^{\prime}$ la contrainte effective $(\mathrm{Pa})$. 
D'après les deux équations précédentes, on obtient l'expression qui donne la vitesse de la phase solide dans un dépôt de vase se consolidant sous son propre poids, à savoir (TAN et al. [11]) :

$$
\bar{w}_{s}=-\frac{k}{\rho_{0}}\left[\left(1-\frac{\rho_{0}}{\rho_{s}}\right) C+\frac{1}{g} \frac{\partial \sigma^{\prime}}{\partial z}\right] .
$$

Lorsque le terme correspondant à la contrainte effective peut être négligé, cette équation devient (BEEN [1]) :

$$
\bar{w}_{s}=-\frac{k}{\rho_{0}}\left(1-\frac{\rho_{0}}{\rho_{s}}\right) C .
$$

Enfin, la variation du coefficient de perméabilité avec la concentration, dans un faible domaine de variation de la concentration, est approchée par la loi suivante :

$$
k=A_{1} \exp \left(-A_{2} \frac{C}{\rho_{s}}\right)
$$

où $A_{1}\left(\mathrm{~m} \mathrm{~s}^{-1}\right)$ et $A_{2}$ sont des paramètres propres à chaque vase.

Cette loi de variation de $k$ (13) a été vérifiée expérimentalement pour une vase molle de l'estuaire de la Loire $\left(W_{L}=150 \%\right)$ ayant des concentrations comprises entre 108 et $330 \mathrm{~kg} \mathrm{~m}^{-3}$ (SANCHEZ et GROVEL [8]); les valeurs des paramètres sont : $A_{1}=0,005 \mathrm{~m} \mathrm{~s}^{-1}$ et $A_{2}=110$.

\section{Comportement de la vase}

Si la contrainte appliquée sur un matériau ne dépasse pas environ $1000 \mathrm{kPa}$, son comportement est plastique (TERZAGH [12]), ce qui a pour conséquence que lorsque l'on enlève la contrainte appliquée, ce matériau ne gonfle pas.

Etant donné que le tassement de la vase est étudié dans un domaine de faibles contraintes de l'ordre de $1 \mathrm{kPa}$, son comportement est considéré plastique.

La transition entre un état de contraintes effectives nulles correspondant à des concentrations inférieures à $C_{0}$ et un état de contraintes effectives finies pour des concentrations supérieures, peut être simulée, dans le cas d'une consolidation unidirectionnelle (frettage latéral), par la loi de comportement à la compressibilité qui suit (SANCHEZ et GROVEl [8]) :

$$
\begin{array}{ll}
\sigma^{\prime}=\left(\frac{C-C_{0}}{B_{1}}\right)^{1 / B_{2}} \text { pour : } C \geqslant C_{0} \\
\sigma^{\prime}=0 & \text { pour : } C<C_{0}
\end{array}
$$

où $B_{1}\left(\mathrm{~kg} \mathrm{~m}^{-3} \mathrm{~Pa}^{-\mathrm{B}_{2}}\right)$ et $B_{2}$ sont des paramètres propres à chaque vase.
D'une manière générale, dans un domaine de faibles contraintes, la valeur de $B_{2}$ est comprise ente 0 et 1 , ce qui confère à cette loi de comportement les propriétés suivantes :

$$
\sigma^{\prime}=0 \text { et } \frac{\partial \sigma^{\prime}}{\partial C}=0 \text { pour : } C \leqslant C_{0} .
$$

Les paramètres de la loi de comportement compressible (14) peuvent être déterminés en laboratoire, soit par la mesure directe du profil vertical de la concentration en fin de consolidation, soit par la mesure de l'épaisseur finale dans plusieurs colonnes de tassement.

Dans le deuxième cas il s'agit de minimiser l'erreur existant entre la hauteur finale mesurée et la hauteur finale résultant de la propre loi de comportement. Une démarche pratique consiste à subdiviser le dépôt en un grand nombre $N c$ de sous-couches d'épaisseur initiale $\mathrm{H}_{0} / \mathrm{Nc}$ constante ( $f$ ig. 3) ; ainsi, la contrainte effective agissant au milieu de la sous-couche $j$, est [9] :

$$
\sigma_{j}=\left[1-\frac{\rho_{0}}{\rho_{s}}\right] g C_{i} \frac{H_{0}}{N c}\left(N c-j+\frac{1}{2}\right)
$$

où $C_{i}$ est la concentration initiale dans le colonne de consolidation $\left(\mathrm{kg} \mathrm{m}^{-3}\right)$ et $H_{0}$ la hauteur initiale de la colonne de consolidation $(\mathrm{m})$.

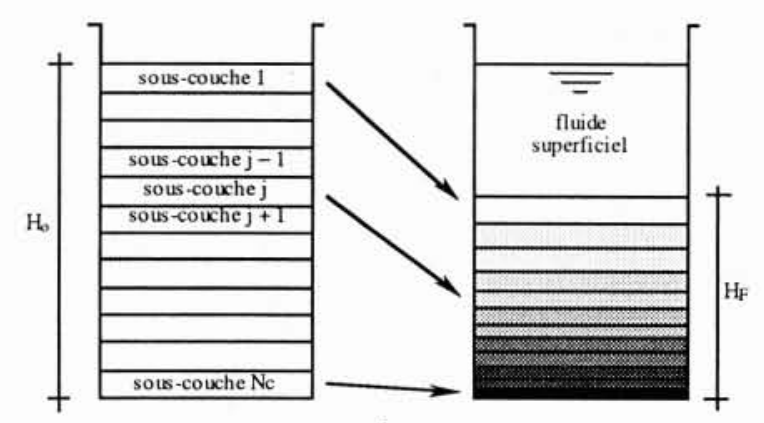

3. Diagramme illustrant le principe d'évaluation de la hauteur finale d'un dépôt de vase selon la loi de comportement à la compressibilité (13).

Etant donné qu'en fin du processus de consolidation $\sigma^{\prime}=\sigma$, la concentration finale $C_{F j}$ de la sous-couche $j$, selon la loi de comportement du matériau (14), est :

$$
C_{F j}=C_{0}+B_{1} \sigma_{j}^{B_{2}} \text { avec : } C_{F j}>C_{i} .
$$

L'épaisseur finale du dépôt $H_{F}$ est donc donnée par:

$$
H_{F}=\sum_{j=1}^{N c} \frac{H_{0}}{N c} \frac{C_{i}}{C_{F j}} .
$$

Ce procédé pour déterminer les paramètres de la loi de comportement à la compressibilité de la vase (14), par 
minimisation de l'erreur existant entre les hauteurs finales mesurées dans plusieurs colonnes de consolidation et celles résultant de la propre loi de comportement (18), est justifié lorsque l'on accepte un même comportement pour l'ensemble des colonnes.

\section{Modèle « iso-concentration » multicouche de tassement des vases}

Une discrétisation « iso-concentration " (fig. 4) permet de simplifier considérablement l'équation de continuité de la phase solide (2) dans le cas de sédimentation d'une suspension, lorsque la vitesse de la phase solide est reliée exclusivement à la concentration par une fonction continue [9].

Dans cette discrétisation des couches de vase en état de suspension, la variation dans le temps de la masse $\boldsymbol{M}_{i}$ (variable d'état, de la couche de vase en état de suspension) est régie par une équation qui s'écrit :

$$
\frac{\partial M_{i}}{\partial t}=Q_{s / C_{i+1}}-Q_{s / C_{i}}, \quad \text { avec : } M_{i} \geqslant 0
$$

pour :

$$
i=1,2,3, \ldots, n
$$

où $M_{i}$ est la masse à l'intérieur de la sous-couche $i$ qui a une concentration comprise entre $C_{i}$ et $C_{i+1}\left(C_{i}<C_{i+1}\right)$ et $Q_{s / C_{i}}$ le débit net à travers l'interface $i$ de concentration $C_{i}$ constante dans le temps.

Lorsque la vitesse de la phase solide est fonction unique de la concentration, la valeur de $Q_{s / C_{i}}$ est constante pour chaque concentration $C_{i}$ et donnée par :

$Q_{s / C_{i}}=-\bar{w}_{s}\left(C_{i}\right) \times C_{i}+C_{i} \times\left.\frac{\mathrm{d}\left[\bar{w}_{s}(C) \times C\right]}{\mathrm{d} C}\right|_{C=C_{i}}$

pour :

$$
i=1,2,3, \ldots, n
$$

où $\bar{w}_{s}$ est la vitesse de la phase solide de la suspension, approchée par (3) et (4).

L'équation (20) est formulée en utilisant les caractéristiques de l'équation (2) pour $\bar{w}_{s}$ fonction unique de la concentration (COURANT et HILBERT [3]). En coordonnées spatio-temporelles, les caractéristiques associées à chaque concentration sont des lignes de pente $W$ constante donnée par:

$$
W\left(C_{i}\right)=\left.\frac{\mathrm{d}\left[\bar{w}_{s}(C) \times C\right]}{\mathrm{d} C}\right|_{c=c_{i}}
$$

Le long de chaque ligne caractéristique la concentration est constante ( $\mathrm{KYNCH}[5]$ ).

L'intégration directe de l'équation (19) conduit à une variation linéaire par rapport au temps de la masse comprise entre deux concentrations fixes. Ceci est valable pour toutes les sous-couches de la suspension, sauf pour la sous-couche en surface, laquelle peut être affectée par

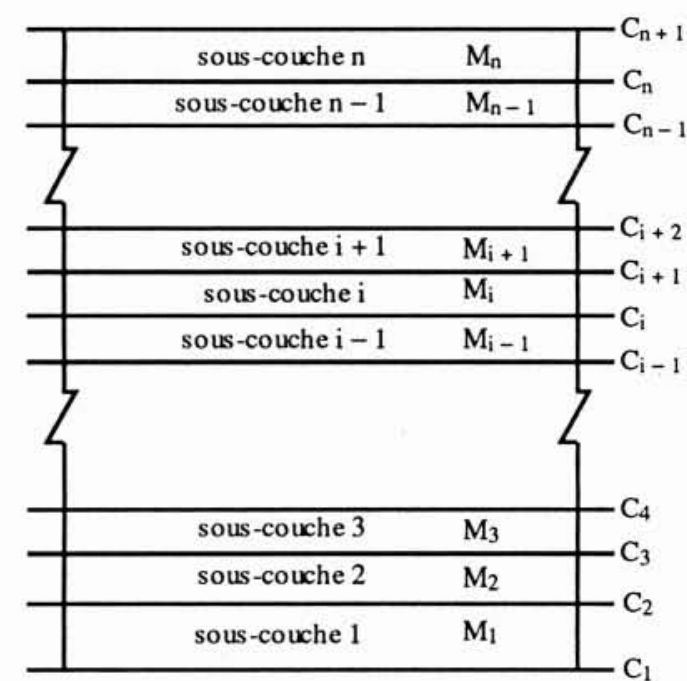

4. Discrétisation utilisée dans le modèle " iso-concentration " multicouche de tassement des vases.

un débit solide d'échange de masse $Q_{D}$ avec la phase fluide superficielle

$$
Q_{s / C_{n+1}}=Q_{D} .
$$

Ce modèle " iso-concentration " de sédimentation peut se généraliser au cas du tassement par consolidation lorsque l'hypothèse suivante est satisfaite :

Hypothèse : L'influence du gradient de contrainte effective sur la vitesse de la phase solide (11) est négligeable.

Dans la pratique, l'hypothèse précédente est partiellement satisfaite dans un domaine de faibles contraintes effectives lorsque la transition entre les phénomènes de sédimentation et de consolidation se fait graduellement.

Cette hypothèse permet d'écrire pour les dépôts de vase en présence des contraintes effectives :

pour $\sigma>\sigma^{\prime}$ :

$Q_{s / C_{i}}=-\bar{w}_{s}\left(C_{i}\right) \times C_{i}+C_{i} \times\left.\frac{\mathrm{d}\left[\bar{w}_{s}(C) \times C\right]}{\mathrm{d} C}\right|_{C=C_{i}}$

pour $\sigma \leqslant \sigma^{\prime}$ :

$$
Q_{s / C_{i}}=0
$$

où $\bar{w}_{s}$ est la vitesse de la phase solide dans le dépôt, donnée par l'équation (12) avec: $\partial \sigma^{\prime} / \partial z=0$.

Ces équations (23) impliquent que l'influence du gradient des contraintes effectives sur la vitesse de la phase solide (11) à l'intérieur d'un dépôt est négligée, mais que le tassement s'arrête pour $\sigma=\sigma^{\prime}$. 
Finalement, la figure 5 montre une courbe caractéristique de variation avec la concentration du débit $Q_{s i C}$, laquelle est construite en considérant une égalité des $k$ et $k^{\prime}$, soit d'après leurs lois de variation respectives (4) et (13) :

$$
A_{1}=A_{3} \quad \text { et } \quad A_{2}=A_{4} \text {. }
$$

Dans la mesure où les lois de variation de $k$ et de $k^{\prime}$, avec des paramètres $A_{1}, A_{2}, A_{3}$ et $A_{4}$ sont justifiées pour une vase donnée, cette courbe est valable dans tous les cas pour une suspension et, lorsque l'influence du gradient de contrainte effective sur la vitesse de la phase solide est négligeable, elle est valable aussi pour les dépôts de vase.

L'interprétation de la figure 5 avec le modèle « isoconcentration " de tassement (19) implique que dans un cours d'eau, pour un débit solide de dépôt donné (ordonnée), l'abscisse correspondante selon la courbe $Q_{s / C}$ donne la concentration $C>\left(2+2^{1 / 2}\right) C_{\bar{w}_{s \max }}$ au-dessus de laquelle la vase s'accumule dans le fond.

\section{Débit solide $\left(\mathrm{kg} \mathrm{m}^{-2} \mathrm{~s}^{-1}\right)$}

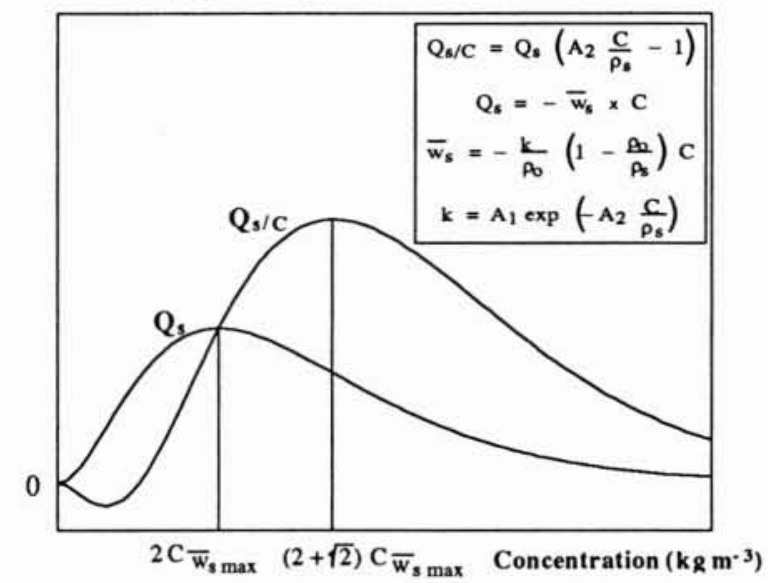

5. Courbe caractéristique de $Q_{s / C}$ fonction de la concentration, associée aux suspensions par la relation $\bar{w}_{s}(C)$ (3) et (4) et aux dépôts par la loi de Darcy modifiée par Scheidegger (7) avec la loi de variation de $k$ (12) lorsque $\partial u / \partial z \approx \partial \sigma / \partial z$.

\section{Conclusions}

1. La relation (3) reliant la vitesse de sédimentation à la concentration d'une vase en état de suspension permet l'assemblage des modèles de sédimentation et de consolidation, car pour $C=C_{0}, k^{\prime}$ est assimilé au coefficient de perméabilité défini selon la loi de Darcy modifiée par SCHEIDEGGER.

Ainsi, pour $C=C_{0}$, la relation (3) s'identifie à l'équation donnant la vitesse de la phase solide dans un dépôt à drainage superficiel (11) et (12).
D'autre part, la loi de variation de $k^{\prime}(4)$, permet de simuler la vitesse de sédimentation à seulement deux paramètres. Cette loi est valable sur pratiquement tout le domaine des concentrations pour lequel une vase est en état de suspension.

Pour certaines vases, une amélioration de la corrélation de la loi $\bar{w}_{s}(C)$ peut être obtenue en modifiant la loi de variation de $k^{\prime}(4)$.

2. Le modèle «iso-concentration" multicouche de tassement des vases constitue un modèle simple qui s'accorde avec l'expérience dans le cas des suspensions ( $\bar{w}_{s}$ liée à $C$ par une fonction continue) et qui rapproche les principes de la théorie unidirectionnelle de consolidation des dépôts sous l'hypothèse que l'effet qu'a le gradient des contraintes effectives sur la vitesse de la phase solide est négligeable.

Dans la pratique, cette hypothèse est partiellement satisfaite dans un domaine de faibles contraintes effectives, lorsque la transition entre l'état de contraintes effectives nulles correspondant à une suspension et l'état des contraintes effectives finies correspondant à un dépôt, se fait graduellement avec l'augmentation de la concentration.

3. Le modèle "iso-concentration" combiné avec les lois de variation de $k$ et de $k^{\prime}$ permet de détecter la concentration au-dessus de laquelle la vase s'accumule dans le fond pour former éventuellement une crème de vase au fond des cours d'eau.

Cette concentration est le résultat des propriétés colloïdales de la vase exprimées par la valeur des paramètres et de l'action hydrodynamique intervenant sur le débit de dépôt $Q_{D}$.

Ce critère est valable dans tous les cas où la concentration à partir de laquelle la vase s'accumule dans le fond correspond à une suspension. Dans le cas où cette concentration correspond à un dépôt, ce critère est conditionné par la validité de l'hypothèse selon laquelle l'effet de $\partial \sigma^{\prime} / \partial z$ sur $\bar{w}_{s}(11)$ est négligeable.

4. Pour une vase molle donnée, la variation avec la concentration de $k^{\prime}$ (4) (SANCHEZ [9]) de même que celle de $k$ (13) (SANCHEZ et GRovel [8] et TAN et al. [11]) suivent généralement une loi exponentielle décroissante dans un domaine limité de variations de $C$.

Une loi variable aussi bien pour $k^{\prime}$ que pour $k$ est la suivante :

$$
k^{\prime}=k=A_{5} \exp \left(-A_{6}\left(\frac{C}{\rho_{s}}\right)^{A^{7}}\right)
$$

où $A_{5}, A_{6}$ et $A_{7}$ sont des paramètres propres à chaque vase. Des valeurs typiques de $A_{7}$ sont comprises entre 0,5 et 1 .

Cette loi, vérifiée pour une vase de l'estuaire de la Loire [9], permet la modélisation du processus général de tassement des vases molles. Dans ce cas, les paramètres $A_{5}, A_{6}$ et $A_{7}$ sont valables aussi bien pour une vase en état de suspension que pour une vase en état de dépôt et ceci pour des contraintes effectives faibles. 


\section{Références bibliographiques}

[1] BEEN K. - - Stress strain behaviour of $\dot{a}$ cohesive soil deposited under water ». Thesis presented to the University of Oxford, at Oxford, England, in partial fulfillment of the degree of Doctor of philosophy, 1980.

[2] Carrier W.D., Bromwell L.G. and Somogy Frank. - «Design capacity of slurried mineral waste ponds ", Journal of Geotechnical Engineering, ASCE, vol. 109, n' 5, May, 1983, pp. 699-716.

[3] Courant R. and HILBERT D. - Methods of mathematical physics, vol. 2, Interscience, New York, 1961.

[4] Gibson R.E., England G.L. and Hussey M.J.L. - « The theory of one-dimensional consolidation of saturated clays. I. Finite nonlinear consolidation of thin homogeneous layers $"$, Geotechnique, vol. 17, n*3, 1967, pp. 261-273.

[5] KYNCH G.F. - «A theory of sedimentation ", Faraday Society Transactions, vol. 48, 1952, pp. 166-176.

[6] Merta A.J. - « Characterization of cohesive sediment properties and transport processes in estuaries ", Estuarine Cohesive Sediment Dynamics, edited by Mehta, SpringerVerlag, New York, 1986, pp. 290-325.

[7] Migniot C. - « Tassement et rhéologie des vases ", $\mathrm{La}$ Houille Blanche, $\mathrm{n}^{\text {os }} 1$ et $2,1989$.

[8] Sanchez M. et Grovel A. - " Consolidation de la vase sous contraintes variables", Journées Nationales Génie Civil-Génie Côtier, Nantes, 1992.

[9] SANChez M. - Modélisation dans un estuaire à marée. Rôle du bouchon vaseux dans la tenue des sols sous-marins, Thèse présentée à l'Université de Nantes en vue de l'obtention du titre de Docteur, Nantes, Juillet 1992.

[10] Scheidegger A.E. - The physics of flow through porous media, Macmillan, New York, 1957.

[11] TAN T.S., Yong K.Y., LeONG E.C. and LeE S.L. - «Sedimentation of clayey slurry », Journal of Geotechnical Engineering, vol. 116, n6, June 1990, pp. 885-898.

[12] Terzaghi K. and Peck R.B. - Mécanique des sols appliquée, Ed. Dunod, Paris, 1961.

[13] Thorn M.F.C. - - Physical processes of siltation in tidal chanels ", Proceedings Hydraulic Modelling Applied to Maritime Engineering Problems, I.C.E., London, 1981, pp. 47-55. 